九州大学学術情報リポジトリ

Kyushu University Institutional Repository

\title{
Symmetric Designs for Exploring Response Surfaces
}

Uranishi, Hisao

General Education Department, Kyushu University

https://doi.org/10.5109/13004

出版情報: 統計数理研究. 10 (3/4), pp. 17-30，1962-10. Research Association of Statistical Sciences

バージョン :

権利関係 : 


\title{
SYMMETRIC DESIGNS FOR EXPLORING RESPONSE SURFACES
}

\author{
By \\ Hisao URANISI \\ (Recieved February 13th, 1962)
}

1. Introduction and Summary. A functional relationship

$$
\eta=\varphi\left(\xi_{1}, \xi_{2}, \cdots, \xi_{k}\right)=\varphi(\xi)
$$

is assumed to exist between a response $\eta$ and $k$ continuous variables $\xi_{1}, \xi_{2}$, $\cdots, \xi_{k}$. To elucidate certain aspects of this relationship, measurements of $\eta$ are to be made for each of $N$ combinations of the levels of the variables $\xi_{u}^{\prime}=\left(\xi_{1 u}, \xi_{2 u}, \cdots, \xi_{k u}\right), u=1, \cdots, N$. We assume that the function $\varphi(\xi)$ can be adequately represented by a polynomial of 2 nd degree within a limited region $\Re$ of immediate interest in the space of the variables. We restrict every experimental point $\xi$ to be in the $k$-dimensional interval $a_{i} \leq \xi_{i} \leq b_{i}$, $(i=1,2, \cdots, k)$, which lies in the region $\Re$.

Putting $x_{i}=2\left(b_{i}-a_{i}\right)^{-1}\left[\xi_{i}-\left(a_{i}+b_{i}\right) / 2\right],(i=1,2, \cdots, k)$, every experimental point must lie in the interval

$$
-1 \leq x_{i} \leq 1, \quad(i=1,2, \cdots, k),
$$

and the response can be represented by

$$
\eta(\mathfrak{x})=\beta_{0}+\sum_{1 \leq i \leq k} \beta_{i} x_{i}+\sum_{1 \leq i \leq j \leq k} \beta_{i j} x_{i} x_{j}
$$

The problem of experimental design considered is the choice of the design matrix $D$ of $N$ rows and $k$ columns whose $u$-th row is $\mathfrak{X}_{u}^{\prime}=\left(x_{1 u}, x_{2 u}\right.$, $\left.\cdots, x_{k u}\right)$, for $u=1, \cdots, N$, which specifies the levels of the variables to be made in each of the $N$ trials. Here we assume that the design matrix $D$ must satisfy the following conditions:

for any integer $\alpha_{i} \geq 0$ such that $\sum_{i=1}^{k} \alpha_{i} \leq 4$, we have

$$
N^{-1} \sum_{u=1}^{N} x_{1 u}^{\alpha_{1}} x_{2 u}^{\alpha_{2}} \cdots x_{k u}^{\alpha k}=\lambda\left(\alpha_{1}, \alpha_{2}, \cdots, \alpha_{k}\right),
$$

if all of the $\alpha_{i}$ are even, and otherwise it is equal to zero, where $\lambda\left(\alpha_{1}, \alpha_{2}\right.$, $\left.\cdots, \alpha_{k}\right)$ is a constant for all permutations of $\alpha_{1}, \alpha_{2}, \cdots, \alpha_{k}$, but it can be different for the different partition of $\alpha=\sum_{i=1}^{k} \alpha_{i}$.

Let us put henceforth

$$
\begin{aligned}
& \lambda_{2}=\lambda(2,0, \cdots, 0)=\lambda(0.2,0, \cdots, 0)=\cdots=\lambda(0, \cdots, 0,2), \\
& \lambda_{3}=\lambda(2,2,0, \cdots, 0)=\cdots=\lambda(0, \cdots, 0,2,2), \\
& \lambda_{4}=\lambda(4,0, \cdots, 0)=\lambda(0,4,0, \cdots, 0)=\cdots=\lambda(0, \cdots, 0,4) .
\end{aligned}
$$


A $k$-dimensional design of order 2 whose design matrix $D$ satisfies the conditions (1.3) will be called a symmetric design of order 2. For the estima bility of all the coefficients in (1.2), any 2 nd order symmetric design must satisfy the relations

$$
\lambda_{2}>0, \quad 0<\lambda_{3}<\lambda_{4}, \lambda_{4}+(k-1) \lambda_{3}-k \lambda_{2}^{2}>0,
$$

as we shall show in section 2 .

Of course a symmetric design having $\lambda_{4}=3 \lambda_{3}$ is a rotatable design of order 2. But we do not assume the rotatability for the present. When we assume (1.3) for any set of $k$ non-negative integers $\alpha_{i}$ satisfying the condition $\sum_{i=1}^{k} \alpha_{i} \leq 2 m$, instead of the condition $\sum_{i=1}^{k} \alpha_{i} \leq 4$, the corresponding designs may be called the $m$-th order symmetric designs. It can be readily seen that if the conditions (1.3) hold true for quite arbitrary set of $k$ nonnegative integers $\alpha_{1}$, the simultaneous moment generating function $G\left(\theta_{1}, \theta_{2}\right.$, $\ldots, \theta_{k}$ ) of the columns of the corresponding design matrix $D$ is a symmetric and even function with respect to $\theta_{1}, \theta_{2}, \cdots, \theta_{k}$. Therefore, the design is symmetric with respect to the coordinate axes of the variables $x_{1}, x_{2}, \cdots, x_{h}$. For any linear model, such as (1.2), in which there are $L$ unknown coe-f ficients, the $N$ equations at the $N$ experimental points may be written in an obvious matrix notation as

$$
\eta=X \boldsymbol{8},
$$

where the $N \times L$ matrix $X$ is called the matrix of independent variables. If the observed values found at the $N$ experimental points are represented by a vector $Y$ and

$$
\Im(Y)=\eta, \quad \Im(Y-\eta)(Y-\eta)^{\prime}=I_{N} \sigma^{2}
$$

then, on the supposition that the mathematical model (1.2) exactly represent the true situation, the "least square" estimate $B$ of the $\beta$ is given by

$$
B=\left(X^{\prime} X\right)^{-1} X^{\prime} Y .
$$

The "least square" estimate $\hat{\eta_{D}}(\boldsymbol{t})$ of the response $\eta(\boldsymbol{t})$ for each assigned $\boldsymbol{t}$ is given by

$$
\hat{\eta}_{D}(\boldsymbol{t})=\tau^{\prime} B
$$

where

$$
\begin{aligned}
\boldsymbol{t}^{\prime} & =\left(t_{1}, t_{2}, \cdots, t_{k}\right), \\
\tau^{\prime} & =\left(1, t_{1}, t_{2}, \cdots, t_{k}, t_{1}^{2}, t_{2}^{2}, \cdots, t_{k}^{2}, t_{1} t_{2}, \cdots, t_{k-1} t_{k}\right) .
\end{aligned}
$$

The standardized variance of $\hat{\eta}_{D}(\boldsymbol{t})$ is given by

$$
N \sigma^{-2} \vee\left[\hat{\eta}_{D}(\boldsymbol{t})\right]=N \tau^{\prime}\left(X^{\prime} X\right)^{-1} \tau,
$$

which becomes, adopting the assumption (1.3) for the 2nd order symmetric designs, 


$$
\begin{aligned}
& \left.\frac{N}{\sigma^{2}} \bigvee\left[\hat{\eta}_{D}(\boldsymbol{t})\right]=1+\frac{\rho^{2}}{\dot{\lambda}_{2}}+\frac{(k-1) \rho^{4}}{2 k \lambda_{3}}+\frac{\left(\rho^{2}-k \lambda_{2}\right)^{2}}{k\left[\lambda_{4}+(k-1) \lambda_{3}-k \lambda_{2}^{2}\right.}\right] \\
& +\frac{3 \lambda_{3}-\lambda_{4}}{2 \lambda_{3}\left(\lambda_{4}-\lambda_{3}\right)}\left(\sum t_{i}^{4}-\frac{\rho^{4}}{k}\right),
\end{aligned}
$$

where

$$
\rho^{2}=\mid \boldsymbol{t}^{2}=t_{1}^{2}+t_{2}^{2}+\cdots+t_{k}^{2} .
$$

The purpose of this paper is to seek for the 2nd order symmetric design which minimizes the above variance function under the conditions,

$$
x_{i u} \leq 1 \quad \text { and } \quad \rho=\text { constant. }
$$

It will be shown that, for a suitable $N$, the desired symmetric design $D^{*}$ has a design matrix whose elements are \pm 1 or 0 . Section 2 is devoted to preparatory consideration by which to deduce (1.11), which was also proved by Box-Hunter [1], p. 213, and then to give (2.8) and (2.9) for $V_{D}(\rho)$ and $(2.10)$ for $V_{D}^{\prime}(\rho)$ respectively. After this preparation we shall give in section 3 Theorems 3.1 and 3.2 regarding min-max value of the variance function. In section 4 we shall turn to discuss to find the 2nd order symmetric design $D^{*}$ which minimizes under the conditions (1.12) the expected value of the standardized variance, assuming the uniform distribution over

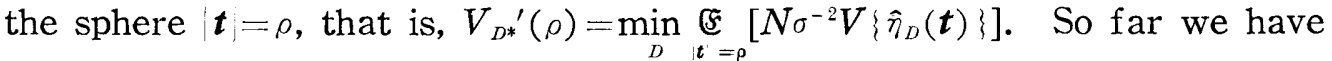
assumed the adequacy of the 2 nd degree response surface, and in section 5 we shall be concerned to give in Teorem 5.1 the alias matrix and the bias due to the third degree terms which might be contained in the true response surface. In section 6 , two examples are discussed in order to show some of practical implications of our resuits obtained in this paper.

2. Deduction of Variance Functions. Let us put

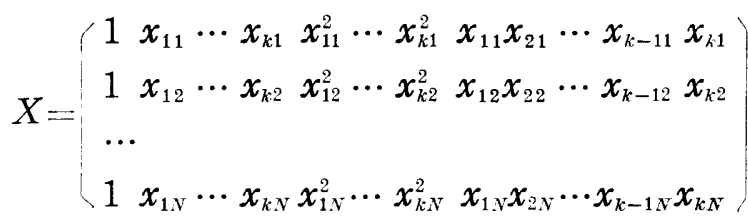

Since the least square estimate $\hat{\eta_{D}}(\boldsymbol{t})$ of $\eta(\boldsymbol{t})$ in (1.2), based on a symmetric design $D$, is given by (1.8) and (1.9), we have

$$
V\left[\hat{\eta}_{D}(\boldsymbol{t})\right]=\tau^{\prime}\left(X^{\prime} X\right)^{-1} \tau \sigma^{2} .
$$

Using the assumptions (1.3) for symmetry, we obtain

$$
\left(\begin{array}{cccccccccc}
1 & 0 & \cdots & 0 & \lambda_{2} & \cdots & \lambda_{2} & 0 & \cdots & 0 \\
0 & \lambda_{2} & \cdots & 0 & 0 & \cdots & 0 & 0 & \cdots & 0 \\
\vdots & \vdots & \ddots & \vdots & \vdots & & \vdots & \vdots & & \vdots
\end{array}\right)
$$




$$
X^{\prime} X=N \begin{array}{cccccccccc}
0 & 0 & \cdots & \lambda_{2} & 0 & \cdots & 0 & 0 & \cdots & 0 \\
\lambda_{2} & 0 & \cdots & 0 & \lambda_{4} & \cdots & \lambda_{3} & 0 & \cdots & 0 \\
\vdots & \vdots & & \vdots & \vdots & \ddots & \vdots & \vdots & & \vdots \\
\lambda_{2} & 0 & \cdots & 0 & \lambda_{3} & \cdots & \lambda_{4} & 0 & \cdots & 0 \\
0 & 0 & \cdots & 0 & 0 & \cdots & 0 & \lambda_{3} & \cdots & 0 \\
\vdots & \vdots & & \vdots & \vdots & & \vdots & \vdots & \ddots & \vdots \\
0 & 0 & \cdots & 0 & 0 & \cdots & 0 & 0 & \cdots & \lambda_{3}
\end{array}
$$

Since $\sigma^{-2} V\left[\hat{\eta}_{D}(\boldsymbol{t})\right]$ is a reciprocal quadratic form of $X^{\prime} X$ with respect to the components of $\tau$, we have

$$
\frac{N}{\sigma^{2}} V\left[\hat{\eta}_{D}(\boldsymbol{t})\right]=\frac{1}{\lambda_{3}} \sum_{i<j} t_{i}^{2} t_{j}^{2}-\frac{1}{\Delta}\left|\begin{array}{cccccccc}
0 & 1 & t_{1} & \cdots & t_{k} & t_{1}^{2} & \cdots & t_{k}^{2} \\
1 & 1 & 0 & \cdots & 0 & \lambda_{2} & \cdots & \lambda_{2} \\
t_{1} & 0 & \lambda_{2} & \cdots & 0 & 0 & \cdots & 0 \\
\vdots & \vdots & \vdots & \ddots & \vdots & \vdots & & \vdots \\
t_{k} & 0 & 0 & \cdots & \lambda_{2} & 0 & \cdots & 0 \\
t_{1}^{2} & \lambda_{2} & 0 & \cdots & 0 & \lambda_{4} & \cdots & \lambda_{3} \\
\vdots & \vdots & \vdots & & \vdots & \vdots & \cdots & \vdots \\
t_{k}^{2} & \lambda_{2} & 0 & \cdots & 0 & \lambda_{3} & \cdots & \lambda_{4}
\end{array}\right|,
$$

where $\Delta$ is a positive definite matrix of order $2 k+1$ obtained from $N^{-1} X^{\prime} X$ deleting its last ${ }_{k} C_{2}$ columns and ${ }_{k} C_{2}$ rows. The last term in the right hand side of (2.4) can be reduced to

$$
1+-\frac{1}{\lambda_{2}} \sum_{i=1}^{k} t_{i}^{2}-\frac{1}{\left|\Delta_{1}\right|}\left|\begin{array}{cccc}
0 & t_{1}^{2}-\lambda_{2} & \cdots & t_{k}^{2}-\lambda_{2} \\
t_{1}^{2}-\lambda_{2} & \lambda_{4}-\lambda_{2}^{2} & \cdots & \lambda_{3}-\lambda_{2}^{2} \\
\vdots & \vdots & \ddots & \vdots \\
t_{k}^{2}-\lambda_{2} & \lambda_{3}-\lambda_{2}^{2} & \cdots & \lambda_{4}-\lambda_{2}^{2}
\end{array}\right|,
$$

where $\Delta_{1}$ is a positive definite matrix of order $k$ with diagonal elements $\lambda_{4}$ $-\lambda_{2}^{2}$ and other elements $\lambda_{3}-\lambda_{2}^{2}$ which appears in the above expression. Since the characteristic roots of $\Delta_{1}$ are $\lambda_{4}-\lambda_{3}$, (replicated $k-1$ times) and $\lambda_{4}+$ $(k-1) \lambda_{3}-k \lambda_{2}^{2}$, and one of its principal axes is $\left(k^{-1 / 2}, k^{-1 / 2}, \cdots, k^{-1 / 2}\right)$, adopting an orthogonal transformation such as

$$
\begin{aligned}
& s_{1}=k^{-1 / 2} \sum_{i=1}^{k}\left(t_{i}^{2}-\lambda_{2}\right), \\
& s_{j}=\sum_{i=1}^{k} \boldsymbol{r}_{i j}\left(t_{i}^{2}-\lambda_{2}\right), \quad(j=2, \cdots, k),
\end{aligned}
$$

we obtain

$$
\frac{N}{\sigma^{2}} V\left[\hat{\eta_{D}}(\boldsymbol{t})\right]=1+\frac{\sum_{i=1}^{k} t_{i}^{2}}{\lambda_{2}}+\frac{\sum_{i<j} t_{i}^{2} t_{j}^{2}}{\lambda_{3}}+\frac{\sum_{j=2}^{k} s_{j}^{2}}{\lambda_{4}-\lambda_{3}}+\frac{s_{1}^{2}}{\lambda_{4}+(k-1) \lambda_{3}-k \lambda_{2}^{2}} .
$$


Since we have, on the $k$-dimensional hypersphere $t=\rho$,

$$
\begin{aligned}
& \sum_{i<j} t_{i}^{2} t_{j}^{2}=\left(\rho^{4}-\sum_{i=1}^{k} t_{i}^{4}\right) / 2, \\
& \sum_{j=2}^{k} s_{j}^{2}=\sum_{i=1}^{k} t_{i}^{4}-\rho^{4} / k,
\end{aligned}
$$

we obtain

$$
\frac{N}{\sigma^{2}} V\left[\hat{\eta}_{D}(\boldsymbol{t})\right]=1+\frac{\rho^{2}}{\lambda_{2}}+\frac{\rho^{4}-\sum t_{i}^{4}}{2 \lambda_{3}}+\frac{\sum t_{1}^{4}-\rho^{4} / k}{\lambda_{4}-\lambda_{3}}+\frac{\left(\rho^{2}-k \lambda_{2}\right)^{2}}{k\left[\lambda_{4}+(k-1) \lambda_{3}-k \lambda_{2}^{2}\right] .}
$$

Since the minimum and the maximum values of $\sum t_{i}^{4}$ on the hypersphere $\sum t_{i}^{2}=\rho^{2}$ are $\rho^{4} / k$ and $\rho^{4}$ respectively, we have the following results.

(a) For the case

$$
\lambda_{3} \leq 3^{-1} \lambda_{4},
$$

$$
V_{D}(\rho)=\max _{i=\rho} \frac{N}{\sigma^{2}} V\left[\hat{\eta}_{D}(\boldsymbol{t})\right]=1+\frac{\rho^{2}}{\lambda_{2}}+\frac{(k-1) \rho^{4}}{2 k \lambda_{3}}+\frac{\left(\rho^{2}-k \lambda_{2}\right)^{2}}{k\left[\lambda_{4}+(k-1) \lambda_{3}-k \lambda_{2}^{2}\right]},
$$

and (b) for the case $\lambda_{3} \geq 3^{-1} \lambda_{4}$

$$
V_{D}(\rho)=\max _{\boldsymbol{t}=\boldsymbol{\rho}} \frac{N}{\sigma^{2}} V\left[\hat{\eta}_{D}(\boldsymbol{t})\right]=1+\frac{\rho^{2}}{\lambda_{2}}+\frac{(k-1) \rho^{4}}{k\left(\lambda_{4}-\lambda_{3}\right)}+\frac{\left(\rho^{2}-k \lambda_{2}\right)^{2}}{k\left[\lambda_{4}+(k-1) \lambda_{3}-k \lambda_{2}^{2}\right]} .
$$

Now, if we associate equal probabilities to all points on the sphere $|\boldsymbol{t}|=\rho$, the expected value $V_{D}{ }^{\prime}(\rho)$ of $N \sigma^{-2} V\left[\hat{\eta}_{D}(\boldsymbol{t})\right]$ becomes

$$
V_{D}^{\prime}(\rho)=1+\frac{\rho^{2}}{\lambda_{2}}+\frac{(k-1) \rho^{4}}{2(k+2) \lambda_{3}}+\frac{2(k-1) \rho^{4}}{k(k+2)\left(\lambda_{4}-\lambda_{3}\right)}+\frac{\left(\rho^{2}-k \lambda_{2}\right)^{2}}{k\left[\lambda_{4}+(k-1) \lambda_{3}-k \lambda_{2}^{2}\right]}
$$

\section{Minimum Value of $V_{D}(\rho)$ for Symmetric Design.}

Under the conditions $\mid x_{i u} \leq 1$ and assumptions (1.3), the moments of the columns of design matrix $D$ must satisfy the inequalities:

$$
\begin{array}{ll}
\text { (i) } & 0<\lambda_{3}<\lambda_{4} \leq \lambda_{2}, \\
\text { (ii) } & \lambda_{4}+(k-1) \lambda_{3}-k \lambda_{2}^{2}>0 .
\end{array}
$$

Since the value of $V_{D}(\rho)$ in (2.8) monotonously decreases as $\lambda_{3}$ increases in the interval $\lambda_{3} \leq 3^{-1} \lambda_{4}$ for any fixed values of $\lambda_{2}$ and $\lambda_{4}, V_{D}(\rho)$ cannot have its minimal point in the interval $\lambda_{3}<3^{-1} \lambda_{4}$. Therefore, it suffices for us to seek the minimum of $V_{L}(\rho)$ in the interval $\lambda_{3} \geq 3^{-1} \lambda_{4}$. In view of the expression (2.9), $V_{D}(\rho)$ decreases as $\lambda_{4}$ increases, for any fixed values of $\lambda_{2}$ and $\lambda_{3}$ and hence it will attain its minimum for $\lambda_{4}=\lambda_{2}$, which is given by

$$
V_{D}(\rho)=1+\frac{\rho^{2}}{\lambda_{2}}+\frac{(k-1) \rho^{4}}{k\left(\lambda_{2}-\lambda_{3}\right)}+\frac{\left(\rho^{2}-k \lambda_{2}\right)^{2}}{k\left[\lambda_{2}+(k-1) \lambda_{3}-k \lambda_{2}^{2}\right]} .
$$


But the equality $\lambda_{4}=\lambda_{2}$ can only happen in the case when all the elements $x_{i u}$ of $D$ are equal to \pm 1 or 0 . Consequently, we should construct our design matrix whose elements are \pm 1 or 0 . Then $\lambda_{2}$ is equal to the ratio of the number of the elements assuming the values \pm 1 in any columns of $D$ devided by $N$, and $\lambda_{3}$ is the relative frequency of rencontres of these \pm 1 elements in any two columns in $D$.

Putting $r=\lambda_{3} / \lambda_{2}$ in the expression (3.2) of $V_{D}(\rho)$, we have

$$
\begin{aligned}
V_{D}(\rho) & =1+\left\{\rho^{2}+\frac{(k-1) \rho^{4}}{k(1-r)}\right\} / \lambda_{2}+\left(\begin{array}{l}
\rho^{2} \\
k
\end{array}-\lambda_{2}\right)^{2} / \lambda_{2}\left\{\frac{1+(k-1) r}{k}-\lambda_{2}\right\} \\
& =\frac{A}{\lambda_{2}}+\frac{B}{C-\lambda_{2}},
\end{aligned}
$$

where we have put

$$
\begin{aligned}
& A=\rho^{2}+\frac{\rho^{4}}{k}\left\{\frac{k-1}{1-r}+\frac{1}{1+(k-1) r}\right\}, \\
& B=\frac{\left\{1+(k-1) r-\rho^{2}\right\}^{2}}{k\{1+(k-1) r\}}, \\
& C=\frac{1+(k-1) r}{k},
\end{aligned}
$$

with the restrictions $1 / 3 \leq r<1$ and $0<\lambda_{2}<C$. For any fixed $r, V_{D}(\rho)$ assumes its minimum at $\lambda_{2}=C_{V} / \bar{A} /(\sqrt{A}+\checkmark \bar{B})$, and we have

$$
\min _{\lambda_{2}} V_{D}(\rho)=(V \bar{A}+\gamma \bar{B})^{2} / C
$$

Let us seek for the minimum of (3.4) as a function of $r$ in the interval $1 / 3 \leq \boldsymbol{r}<1$. Now we have

$$
\frac{d}{d r} \min _{\lambda_{2}} V_{l}(\rho)=\frac{\sqrt{A}+\sqrt{B}}{C^{2}} \cdot \frac{(k-1) \rho^{4}}{k_{V} / \bar{A}}\{ \pm 2 \sqrt{P}-Q\},
$$

where we have put

$$
\begin{aligned}
& P=\frac{1}{k\{1+(k-1) \boldsymbol{r}\}}\left[\frac{1}{\rho^{2}}+\frac{k-1}{k(1-r)}+\frac{1}{k\{1+(k-1) r\}}\right], \\
& Q=\frac{1}{\rho^{2}}+\frac{2(k-1)}{k(1-r)}+\frac{2}{k\{1+(k-1) \boldsymbol{r}\}}-\frac{1}{(1-\boldsymbol{r})^{2}},
\end{aligned}
$$

and either of double signs \pm should be selected according as $1+(k-1) r$ $-\rho^{2} \gtrless 0$. Since we have 


$$
Q^{2}-4 P=\left[\rho^{-2}+2(k-1) / k(1-r)-(1-r)^{-2}\right]^{2}-4 \cdot k^{-7}(1-r)^{-2},
$$

the inequality $Q^{2}-4 P \geqq 0$ gives the solution

$$
r \leq \alpha=\frac{-2 / k+\sqrt{k^{-2}(k-2)^{2}+\rho^{-2}}}{(k-2) / k+\sqrt{k^{-2}(k-2)^{2}+\rho^{-2}}} \text { and } r \geq \beta=\frac{\sqrt{1+\rho^{-2}}}{1+\checkmark 1+\rho^{-2}} \text {. }
$$

But since we observe that

$$
Q>0 \text { at } r=\alpha \text { and } Q<0 \text { at } r=\beta,
$$

we obtain the following results :

(i) For the case $\rho^{2} \leq(k+2) / 3$, where $1 \div(k-1) r-\rho^{2} \geq 0$, the variance function $V_{D}(\rho)$ attains its minimum at $r=\max (\alpha, 1 / 3)$.

(ii) For the case $\rho^{2} \geq k$, where $1+(k-1) r-\rho^{2}<0, V_{D}(\rho)$ attains its minimum at $r=\beta$ which is greater than $1 / 2$.

(iii) For the case $(k+2) / 3<\rho^{2}<k$, the derivative of $V_{D}(\rho)$ becomes discontinuous at $r=\left(\rho^{2}-1\right) /(k-1)$. The minimal point of $V_{\nu}(\rho)$ is given by $\boldsymbol{r}=\alpha$ or $\beta$ or $\left(\rho^{2}-1\right) /(k-1)$ according as $\alpha>\left(\rho^{2}-1\right) /(k-1)$ or $\beta<\left(\rho^{2}-1\right) /$ $(k-1)$ or otherwise.

In this case (iii) we observe that the minimal point $r$ is greater than $1 / 3$. In the case (i), a rotatable design where $r=1 / 3$ can give the minimum of the variance function $V_{D}(\rho)$, when we have $\alpha \leq 1 / 3$. Now, it is to be noted that the inequality $\alpha \leq 1 / 3$ is equivalent to the condition $\rho^{2} \geq 4 k / 3(8-k)>0$, we obtain the following theorems:

Theorem 3.1. Among the k-dimensional symmetric designs $D$ of order 2 under the conditions $\left|x_{i u}\right| \leq 1$, a second order rotatable design can give

\begin{tabular}{|c|c|c|c|}
\hline \multirow{2}{*}{$\rho 2$} & \multirow{2}{*}{$V_{D *}(\rho)$} & \multicolumn{2}{|c|}{$\min -\max \operatorname{design} D^{*}$} \\
\hline & & $\lambda_{2}=\lambda_{4}$ & $r=\lambda_{3} / \lambda_{4}$ \\
\hline 0.2 & 2.0944 & 0.3090 & 0.5528 \\
\hline 0.4 & 2.6649 & 0.3874 & 0.3675 \\
\hline $4 / 9$ & 2.7778 & 0.4000 & $1 / 3$ \\
\hline 0.6 & 3.1667 & 0.4737 & $1 / 3$ \\
\hline 0.8 & 3.5205 & 0.5270 & $1 / 3$ \\
\hline 1.0 & 4.1427 & 0.5848 & $1 / 3$ \\
\hline $4 / 3$ & 5.0000 & 0.6667 & $1 / 3$ \\
\hline 1.5 & 6.0000 & 0.7500 & 0.5000 \\
\hline 2.0 & 11.899 & 0.7101 & 0.5505 \\
\hline 3.0 & 29.856 & 0.6340 & 0.5359 \\
\hline 4.0 & 54.136 & 0.5959 & 0.5279 \\
\hline
\end{tabular}
the min-max solution of (3.6)

Table 1. $V_{D^{*}}(\rho)=\min _{\nu} \max _{i t=\rho} \underset{\sigma^{2}}{N} V\left[\hat{\eta}_{D}(t)\right]$.

$$
k=3
$$

\begin{tabular}{|c|c|c|c|}
\hline$\rho^{2}$ & \multirow{2}{*}{$V_{D^{*}}(\rho)$} & \multicolumn{2}{|c|}{ min-max design $D^{*}$} \\
\hline 0.2 & 2.3068 & 0.2977 & 0.6145 \\
0.4 & 3.1673 & 0.3632 & 0.4870 \\
0.6 & 4.0000 & 0.4000 & 0.4000 \\
0.8 & 4.8400 & 0.4242 & $1 / 3$ \\
1.0 & 5.7120 & 0.4626 & $1 / 3$ \\
$5 / 3$ & 9.0000 & 0.5555 & $1 / 3$ \\
2.0 & 12.000 & 0.6667 & 0.5000 \\
2.5 & 19.874 & 0.6635 & 0.5420 \\
3.0 & 29.856 & 0.6340 & 0.5359 \\
4.0 & 55.833 & 0.5985 & 0.5279 \\
5.0 & 89.818 & 0.5779 & 0.5228 \\
\hline
\end{tabular}




$$
\begin{aligned}
& V_{D^{*}}(\rho)=\min _{D} \max _{t=\rho} N \sigma^{-2} V\left[\hat{\eta}_{D}(\boldsymbol{t})\right], \\
& \text { only when } 4 k / 3(8-k) \leq \rho^{2} \leq(k+2) / 3, \quad(k=-2,3,4,5) .
\end{aligned}
$$

Theorem 3.2. Any 2nd order symmetric design $D$ under the conditions $x_{i u} \leq 1$, cannot give its least square estimate $\hat{\eta}_{D}(\boldsymbol{t})$ of the response $\eta(\boldsymbol{t})$, the maximum standardized variance on the hyper-sphere $t=\rho$, less than the values tabulated in Table 1.

\begin{tabular}{|c|c|c|c|}
\hline \multicolumn{4}{|c|}{$k=5$} \\
\hline 0.5 & 4.2771 & 0.3714 & 0.5319 \\
\hline 1.0 & 7.6518 & 0.4216 & 0.4338 \\
\hline 2.0 & 16.4408 & 0.4574 & 0.3453 \\
\hline 2.25 & 19.080 & 0.4629 & $1 / 3$ \\
\hline 3.0 & 30.000 & 0.6000 & 0.5000 \\
\hline 4.0 & 55.833 & 0.5985 & 0.5279 \\
\hline 5.0 & 89.818 & 0.5779 & 0.5228 \\
\hline
\end{tabular}

\begin{tabular}{|c|c|c|c|}
$k=4$ & \\
\hline 0.5 & 4.0000 & 0.3750 & 0.5000 \\
1.0 & 6.8540 & 0.4271 & 0.3820 \\
1.5 & 9.3406 & 0.4591 & $1 / 3$ \\
2.0 & 14.0000 & 0.5000 & $1 / 3$ \\
3.0 & 29.856 & 0.6340 & 0.5359 \\
4.0 & 55.833 & 05985 & 0.5279 \\
5.0 & 89.818 & 0.5779 & 0.5228 \\
\hline
\end{tabular}

\begin{tabular}{|c|c|c|c|}
\hline 0.5 & 4.6544 & 0.4005 & 0.6322 \\
\hline 1.0 & 9.0305 & 0.4094 & 0.4854 \\
\hline 2.0 & 19.7025 & 0.4529 & 0.4184 \\
\hline 3.0 & 34.789 & 0.4671 & 0.3875 \\
\hline 4.0 & 56.000 & 0.5714 & 0.5000 \\
\hline 5.0 & 89.818 & 0.5779 & 0.5228 \\
\hline
\end{tabular}

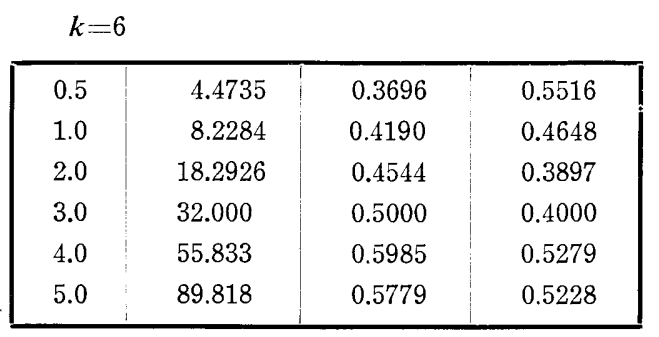

$D^{*}$ is a $k$-dimensional 2 nd order symmetric design matrix with elements $x_{i u}= \pm 1$ or $0,(i=1,2, \cdots, k ; u=1, \cdots N) . \lambda_{2}$ is the relative frequency of \pm 1 . elements in any column in $D^{*} . r$ is the relative frequency of rencontres of \pm 1 elements in any two columns in $D^{*}$.

\section{Minimization of the Expected Variance.}

Since it becomes clear that a rotatable design of order 2 cannot always give the best estimates of the 2 nd degree regression functions, we proceed to seek for a 2nd order symmetric design which may give a best estimate of the response $\eta(t)$. For this purpose, we shall adopt for the criterion the mean value $V_{D}^{\prime}(\rho)$ of the standardized variance $N \sigma^{-2} V\left[\hat{\eta}_{D}(\boldsymbol{t})\right]$ in $(2.10)$. An argument similar to that used in the previous section will give a 2 nd order symmetric design which minimizes $V_{D}^{\prime}(\rho)$ for each assigned $\rho$, if we do not restrict the size $N$ of the design. Under the conditions $\left|x_{i u}\right| \leq 1$ the variance function $V_{D}{ }^{\prime}(\rho)$ in $(2.10)$ decreases as $\lambda_{4}$ increases for any fixed values of $\lambda_{2}$ and $\lambda_{3}$. Therefore, $V_{D}{ }^{\prime}(\rho)$ attains its minimum when we put $\lambda_{4}=\lambda_{2}$, which means that the desired design has all its experimental points at the $k$-dimensionaI lattice points $x_{i u}= \pm 1$ or 0 . 
Putting

$$
\lambda_{4}=\lambda_{2} \text { and } r=\lambda_{3} / \lambda_{4}
$$

the variance function $V_{D}{ }^{\prime}(\rho)$ becomes

$$
\text { (4.1) } \quad V_{j}^{\prime}(\rho)=1+\frac{1}{\lambda_{2}}\left[\rho^{2}+\frac{(k-1) \rho^{4}}{2(k+2) r} \begin{array}{c}
2(k-1) \rho^{4} \\
k(k+2)(1-r)
\end{array}\right]+\frac{\left(k \lambda_{2}-\rho^{2}\right)^{2}}{k_{k} \lambda_{2}\left[1+(k-1) r-k \lambda_{2}\right]} \text {. }
$$

This expression attains its minimum, for any fixed $r$, at $\lambda_{2}=C^{\prime} \checkmark A^{\prime} /\left(\checkmark A^{\prime}\right.$ $\left.+\sqrt{B^{\prime}}\right)$, and we have

$$
\min _{\lambda_{2}} V_{D}^{\prime}(\rho)=\left(\sqrt{A^{\prime}}+\sqrt{B^{\prime}}\right)^{2} / C^{\prime},
$$

where we have put

$$
\begin{aligned}
& A^{\prime}=\rho^{2}+\frac{(k-1) \rho^{4}}{2(k+2) r}+\frac{2(k-1) \rho^{4}}{k(k+2)(1-r)}+k[1+(k-1) r] \\
& B^{\prime}=\left[1+(k-1) r-\rho^{2}\right]^{2} / k[1+(k-1) r], \\
& C^{\prime}=[1+(k-1) r] / k .
\end{aligned}
$$

Differentiating $\min _{\lambda_{2}} V_{D}^{\prime}(\rho)$ in (4.2) with respect to $r$, we have

$$
d r \min _{\lambda_{2}} V_{D}^{\prime}(\rho)=\frac{\sqrt{A^{\prime}+V} \overline{B^{\prime}}}{C^{\prime 2}} \cdot \frac{(k-1) \rho^{4}}{k_{V} / \overline{A^{\prime}}}\left[ \pm 2 / \overline{P^{\prime}}-Q^{\prime}\right]
$$

where we have put

$$
\begin{aligned}
& P^{\prime}=\left[\frac{1}{\rho^{2}}+\frac{k-1}{k(k+2)}\left(\frac{k}{2 r}+\frac{2}{1-r}\right)+\frac{1}{k\{+1(k-1) r\}}\right] / k[1+(k-1) r], \\
& Q^{\prime}=\frac{1}{\rho^{2}}+\frac{2(k-1)}{k(k+2)}\left(\frac{k}{2 r}+\frac{2}{1-r}\right)+\frac{2}{k\{1+(k-1) r\}}-\frac{1}{2(k+2)}\left\{\frac{4}{(1-r)^{2}}-\frac{1}{r^{2}}\right\},
\end{aligned}
$$

the double sings \pm should be selected according as $1+(k-1) r-\rho^{2} \geqslant 0$. We shall try to solve the inequality $\pm 2, \overline{P^{\prime}}-Q^{\prime} \leq 0$ with respect to $r$.

We have in the interval $0<r<1$,

$$
\begin{aligned}
& \left.\frac{d Q^{\prime}}{d r}+\frac{2(k-1)}{k[\hat{1}+(k-1)} \bar{r}\right]^{2}<0, \\
& \frac{d}{d r}\left(Q^{\prime 2}-4 P^{\prime}\right)=2 Q^{\prime}\left\{\frac{d Q^{\prime}}{d r}+\frac{2(k-1)}{k[1+(k-1) r]^{2}}\right\},
\end{aligned}
$$

and

$$
\begin{aligned}
Q^{\prime 2}-4 P^{\prime}= & {\left[\frac{1}{\rho^{2}}+\frac{2(k-1)}{k(k+2)}\left(\frac{k}{2 r}+\frac{2}{1-r}\right)-\frac{1}{2(k+2)}\left\{\frac{4}{(1-r)^{2}}-\frac{1}{r^{2}}\right\}\right]^{2} } \\
& -\frac{8 r^{2}-2 k(1-r)^{2}}{k^{2}(k+2) \boldsymbol{r}^{2}(1-r)^{2}} .
\end{aligned}
$$


By virtue of (4.4), $Q^{\prime}$ decreases from $+\infty$ to $-\infty$ monotonously in the interval $0<r<1$. Therefore, $Q^{\prime}$ has an unique root somewhere at $r=r_{0}$ in the open interval $(0,1)$. Now we observe that

$$
r_{0}>\sqrt{k} /(\sqrt{k}+2) \text {, }
$$

since $Q^{\prime}$ is positive at $r=\sqrt{k} /(V k+2)$. By virtur of (4.4) and (4.5), the expression $Q^{\prime 2}-4 P^{\prime}$ is decreasing in the interval $r<r_{0}$, attains its minimum value which is negative at $r=\boldsymbol{r}_{0}$, and hereafter increases monotonously to $+\infty$. Therefore, the equation $Q^{\prime 2}-4 P^{\prime}=0$ has two roots $r_{1}$ and $r_{2}$ in the interval $0<r<1$, which are

$$
\sqrt{k} /(\sqrt{k}+2)<r_{1}<r_{0}<r_{2},
$$

since $Q^{\prime 2}-4 P^{\prime}$ is positive at $r=\sqrt{k} /(\sqrt{k}+2)$.

Now we shall proceed to give the minimum value of the variance function (4.2) for three exclusive cases:

(i) For the case $\rho^{2} \leq(k+2) / 3$, we have $1+(k-1) r-\rho^{2} \geq 0$ in the interval $1 / 3 \leq r<1$. Therefore, $\frac{d}{d r} \min _{\lambda_{2}} V_{D}{ }^{\prime}(\rho)$ in (4.3)

is always negative in the interval $r<r_{1}$, and is always positive in the interval $r>r_{1}$. The variance function (4.2) attains its minimum at $r=r_{1}$. (ii) For the case $\rho^{2} \geq k$, we have always $1+(k-1) r-\rho^{2}<0$. Therefore, $\frac{d}{d r} \min _{\lambda_{2}} V_{D}{ }^{\prime}\left(d^{\prime}\right)$ is always negative in the interval $r<r_{2}$, and is always positive in the interval $r>r_{2}$. The variance function attains its minimum at $r=r_{2}$.

(iii) For the case $(k+2) / 3<\rho^{2}<k, \frac{d}{d r} \min _{\lambda_{2}} V_{D}{ }^{\prime}(\rho)$ is discontinuous at $r=$ $\left(\rho^{2}-1\right) /(k-1)$. In this case (iii) the minimal point $r^{\prime}$ of $\min _{\lambda_{2}} V_{D}^{\prime}(\rho)$ is given as follows,

a) when $Q^{\prime 2}-4 P^{\prime}<0$ at $r=\left(\rho^{2}-1\right) /(k-1), r^{\prime}=\left(\rho^{2}-1\right) /(k-1)$,

b) when $Q^{\prime 2}-4 P^{\prime} \geq 0$ and $Q^{\prime}>0$ at $r=\left(\rho^{2}-1\right) /(k-1), r^{\prime}=r_{1}$,

c) when $Q^{\prime 2}-4 P^{\prime} \geq 0$ and $Q^{\prime}<0$ at $r=\left(\rho^{2}-1\right) /(k-1), r^{\prime}=r_{2}$.

Throughout all these cases, we observe that the minimal point is greater than $\sqrt{k} /(V \bar{k}+2)$ by virtue of (4.8).

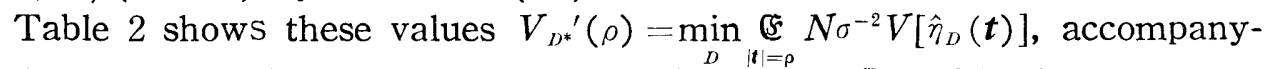
ing the corresponding 2 nd order symmetric desings $D^{*}$ with elements $x_{i u}=$ \pm 1 or 0 .

\section{Alias matrix for 2nd order symmetric designs.}

When the regression function $\eta(\mathfrak{X})$, instead of the expression (1.2), contains 3 rd order terms and is adequately represented by (5.1) 
Table 2. $V_{D^{*}}(\rho)=\min _{D} \underset{t=\rho}{[} N \sigma-2 V\left[\hat{\eta}_{D}(t)\right]$.

\begin{tabular}{|c|c|c|c|}
\hline \multirow{2}{*}{$\rho^{2}$} & \multirow{2}{*}{$V_{L, *^{\prime}}(\rho)$} & \multicolumn{2}{|c|}{$\operatorname{design} D^{*}$} \\
\hline & & $\lambda_{2}=\lambda_{4}$ & $r=\lambda_{3} / \lambda_{4}$ \\
\hline 0.5 & 2.8163 & 0.4649 & 0.5636 \\
\hline 1.0 & 4.0000 & 0.6250 & 0.5000 \\
\hline 1.5 & 5.2500 & 0.7500 & 0.5000 \\
\hline 2.0 & 95327 & 0.7847 & 0.6747 \\
\hline 3.0 & 24.195 & 0.6968 & 0.6650 \\
\hline 4.0 & 45.476 & 0.6564 & 0.6598 \\
\hline 5.0 & 73.366 & 0.6331 & 0.6565 \\
\hline
\end{tabular}

$$
k=3
$$

\begin{tabular}{|c|c:c:c|}
\hline$\rho^{2}$ & \multirow{2}{*}{$V_{l, *^{\prime}(\rho)}$} & \multicolumn{2}{|c|}{ design $D^{*}$} \\
\hline 0.5 & 3.2632 & $\lambda_{2}=\lambda_{4}$ & $r=\lambda_{3} / \lambda_{4}$ \\
1.0 & 5.0997 & 0.4351 & 0.6521 \\
1.5 & 7.1690 & 0.6541 & 0.5944 \\
2.0 & 9.5183 & 0.6929 & 0.5708 \\
2.5 & 13.6856 & 0.7825 & 0.5582 \\
3.0 & 20.720 & 0,7448 & 0.6900 \\
4.0 & 39.096 & 0.7001 & 0.6861 \\
5.0 & 63.210 & 0.6744 & 0.6811 \\
\hline
\end{tabular}

$$
k=4
$$

\begin{tabular}{|l|r|l|l|}
\hline 0.5 & 3.4817 & 0.4263 & 0.6990 \\
1.0 & 5.6726 & 0.5323 & 0.6487 \\
1.5 & 8.2103 & 0.5997 & 0.6269 \\
2.0 & 11.1385 & 0.6478 & 0.6148 \\
2.5 & 14.4704 & 0.6843 & 0.6073 \\
3.0 & 18.500 & 0.7500 & 0.6667 \\
3.5 & 25.905 & 0.7554 & 0.7001 \\
4.0 & 34.741 & 0.7350 & 0.6979 \\
5.0 & 56.267 & 0.7073 & 0.6949 \\
\hline
\end{tabular}

$k=5$

\begin{tabular}{|l|l|l|l|}
\hline 0.5 & 3.5969 & 0.4229 & 0.7289 \\
1.0 & 5.9835 & 0.5233 & 0.6837 \\
1.5 & 8.7851 & 0.5855 & 0.6636 \\
2.0 & 12.0436 & 0.6291 & 0.6524 \\
2.5 & 15.7714 & 0.6617 & 0.6452 \\
3.0 & 19.9736 & 0.6871 & 0.6403 \\
3.5 & 24.653 & 0.7075 & 0.6367 \\
4.0 & 31.563 & 0.7641 & 0.7119 \\
4.5 & 40.791 & 0.7475 & 0.7102 \\
5.0 & 51.193 & 0.7345 & 0.7089 \\
\hline
\end{tabular}

$k=7$

\begin{tabular}{|r|r|r|r|}
\hline 0.5 & 3.6964 & 0.4209 & 0.7662 \\
1.0 & 6.2580 & 0.5171 & 0.7273 \\
1.5 & 9.2985 & 0.5751 & 0.7097 \\
2.0 & 12.8577 & 0.6152 & 0.6997 \\
2.5 & 16.9474 & 0.6448 & 0.6933 \\
3.0 & 21.5725 & 0.6677 & 06888 \\
3.5 & 26.7355 & 0.6859 & 0.6855 \\
4.0 & 32.4377 & 0.7007 & 0.6830 \\
4.5 & 38.680 & 0.7131 & 0.6810 \\
5.0 & 45.463 & 0.7235 & 0.6793 \\
5.5 & 54.267 & 0.7660 & 0.7298 \\
6.0 & 65.332 & 0.7565 & 0.7288 \\
\hline
\end{tabular}


Table 2. (continued).

\begin{tabular}{|c|c|c|c|}
\hline$k=8$ & \multicolumn{3}{|c|}{} \\
\hline$\rho^{2}$ & $V_{D^{*}}(\rho)$ & $\lambda_{2}=\lambda_{4}$ & $r=\lambda_{3} / \lambda^{\circ}$ \\
& & design & $D^{*}$ \\
\hline 0.5 & 3.7163 & 0.4207 & 0.7792 \\
1.0 & 6.3139 & 0.5161 & 0.7424 \\
1.5 & 9.4038 & 0.5732 & 0.7256 \\
2.0 & 13.0252 & 0.6162 & 0.7161 \\
2.5 & 17.1900 & 0.6416 & 0.7099 \\
3.0 & 21.903 & 0.6639 & 0.7056 \\
3.5 & 27.166 & 0.6816 & 0.7024 \\
4.0 & 32.981 & 0.6961 & 0.7000 \\
4.5 & 39.349 & 0.7081 & 0.6980 \\
5.0 & 46.270 & 0.7183 & 0.6965 \\
5.5 & 53.744 & 0.7270 & 0.6952 \\
6.0 & 61.920 & 0.7500 & 0.7143 \\
6.5 & 73.079 & 0.7650 & 0.7371 \\
7.0 & 85.466 & 0.7579 & 0.7364 \\
7.5 & 98.820 & 0.7518 & 0.7358 \\
\hline
\end{tabular}

\begin{tabular}{|c|c|c|c|}
\hline \multirow{2}{*}{$\rho^{2}$} & \multirow{2}{*}{$V_{D^{*}}(\rho)$} & \multicolumn{2}{|c|}{ design $D^{*}$} \\
\hline & & $\lambda_{2}=\lambda_{4}$ & $r=\lambda_{3} / \lambda_{4}$ \\
\hline 0.5 & 3.7265 & 0.4207 & 0.7898 \\
\hline 1.0 & 6.3432 & 05156 & 0.7547 \\
\hline 1.5 & 9.4591 & 0.5723 & 0.7387 \\
\hline 2.0 & 131133 & 0.6112 & 0.7295 \\
\hline 2.5 & 17,3174 & 0.6398 & 0.7236 \\
\hline 3.0 & 22.076 & 0.6618 & 0.7194 \\
\hline 3.5 & 27.392 & 0.6792 & 0.7163 \\
\hline 4.0 & 33.266 & 0.6935 & 0.7140 \\
\hline 4.5 & 39.700 & 0.7053 & 0.7121 \\
\hline 5.0 & 46.693 & 0.7154 & 0.7106 \\
\hline 5.5 & 54.246 & 0.7239 & 0.7093 \\
\hline 6.0 & 62.359 & 0.7313 & 0.7082 \\
\hline 6.5 & 71.033 & $0 \cdot 7377$ & 0.7073 \\
\hline 7.0 & 81.252 & 0.7722 & 0.7444 \\
\hline 7.5 & 93.960 & 0.7660 & 0.7438 \\
\hline
\end{tabular}

$$
\eta(\mathfrak{X})=\beta_{0}+\sum_{1 \leq i \leq h} \beta_{i} x_{i}+\sum_{i \leq j} \beta_{i j} x_{i} x_{j}+\sum_{i \leq j \leq h} \beta_{i j h} x_{i} x_{j} x_{h},
$$

or $\eta(\mathfrak{X})=X \beta+X_{1} \beta_{1}$ for short, the least square estimate $B$ of $\beta$ in (1.8) is biased for we have

$$
\xi(B)=\beta+(X X)^{-1} X^{\prime} X_{1} \beta_{1}=\beta+A \beta_{1} .
$$

The 'alias matrix' $A=\left(X^{\prime} X\right)^{-1} X^{\prime} X_{1}$ will take the following simple form (5.7) if we assume for the design matrix $D$, in addition to the 2nd order symmetry, that the fifth order moments of the columns of $D$ are all zero.

By the rearrangement of the independent variables, the $u$-th rows of $X$ and $X_{1}$ can be respectively represented as follows,

$$
\begin{aligned}
& \left(1, x_{1 u}^{2}, \cdots, x_{k u}^{2}, x_{1 u}, \cdots, x_{k u}, x_{1 u} x_{2 u}, x_{1 u} x_{3 u}, \cdots, x_{k-1 u} x_{k u}\right), \\
& \left(x_{1 u}^{3}, x_{1 u} x_{2 u}^{2}, \cdots, x_{1 u} x_{k u}^{2}, x_{2 u}^{3}, x_{2 u} x_{1 u}^{2}, x_{2 u} x_{3 u}^{2}, \cdots, x_{3 u}^{3}, \cdots, x_{1 u} x_{2 u} x_{3 u}, \cdots\right)
\end{aligned}
$$

where

$$
u=1, \cdots, N \text {. }
$$

Using the assumptions for symmetry (1.3), we have

$$
{ }_{N}^{1} X^{\prime} X=\left(\begin{array}{cc}
\Delta & 0 \\
0^{\prime} E
\end{array}\right), \quad \text { where } \Delta=\left(\begin{array}{cccc}
1 & \lambda_{2} & \cdots & \lambda_{2} \\
\lambda_{2} & \lambda_{4} & \cdots & \lambda_{3} \\
\vdots & \vdots & \ddots & \vdots \\
\lambda_{2} & \lambda_{3} & \cdots & \lambda_{4}
\end{array}\right),
$$


$\Delta$ is a $k+1$ by $k+1$ symmetric matrix,

0 is a $k+1$ by ${ }_{k+1} C_{2}$ zero matrix and $0^{\prime}$ is its transform,

$E$ is a diagonal matrix of order ${ }_{k+1} C_{2}$ whose diagonal elements are $\lambda_{2}$ followed by $\lambda_{3}$ replicated ${ }_{k} C_{2}$ times.

Using the assumptions that fifth order moments of the design matrix $D$ are all zero in addition to the assumptions for symmetry (1.3), we have

$$
\stackrel{1}{N} X^{\prime} X_{1}=\left(\begin{array}{cccc}
0_{1} & \cdots & 0_{1} & 0_{3} \\
E_{1} & \cdots & E_{k} & 0_{4} \\
0_{2} & \cdots & 0_{2} & 0_{5}
\end{array}\right.
$$

where $0_{1}, 0_{2}, 0_{3}, 0_{4}$ and $0_{5}$ are zero matrices whose sizes are respectively $(k+1) \times k,{ }_{k} C_{2} \times k,(k+1) \times{ }_{k} C_{3}, k \times{ }_{k} C_{3}$ and ${ }_{k} C_{2} \times{ }_{k} C_{3} . \quad E_{i}$ is a $k \times k$ square matrix whose elements are all zero except the $i$-th row which is $\left(\lambda_{4}, \lambda_{3}, \cdots\right.$, $\left.\lambda_{3}\right)$. $(i=1,2, \cdots, k)$. Therefore, we obtain the following

Theorem 5.1. Under the assumption (5.1) of this paragraph, the alias matrix is given by

$$
A=\frac{1}{\lambda_{2}}\left(\begin{array}{cccc}
0_{1} & \cdots & 0_{1} & 0_{3} \\
E_{1} & \cdots & E_{k} & 0_{4} \\
0_{2} & \cdots & 0_{2} & 0_{5}
\end{array}\right),
$$

and the bias of the least square estimate $\hat{\eta}_{D}(\boldsymbol{t})$ is given by

$$
\left(\tau^{\prime} A-\tau_{1}^{\prime}\right) \beta_{1}=\frac{1}{\lambda_{2}} \sum_{i=1}^{k} t_{i}\left(\lambda_{4} \beta_{i i i}+\lambda_{3} \sum_{j \neq i} \beta_{i j, j}\right)-\sum_{i \leq j \leq h} \beta_{i j h} t_{i} t_{j} t_{h} .
$$

6. Numerical Examples. For an example, let us compare following two 3 -factor designs of experiments having the same size $N=15$.

$$
D_{1}=\left(\begin{array}{rrr}
1 & 1 & 1 \\
1 & 1 & -1 \\
1 & -1 & 1 \\
1 & -1 & -1 \\
-1 & 1 & 1 \\
-1 & 1 & -1 \\
-1 & -1 & 1 \\
-1 & -1 & -1 \\
1 & 0 & 0 \\
-1 & 0 & 0 \\
0 & 1 & 0 \\
0 & -1 & 0 \\
0 & 0 & 1 \\
0 & 0 & -1 \\
0 & 0 & 0
\end{array}\right)
$$

$$
\begin{aligned}
& \lambda_{2}=\lambda_{4}=2 / 3, \\
& r=\lambda_{3} / \lambda_{4}=0.8 \text {, } \\
& V_{D_{1}}{ }^{\prime}(\rho)=\frac{13}{3}-\frac{11}{6} \rho^{2}+\frac{77}{24} \rho^{4}, \\
& \begin{array}{c|r}
\rho^{2} & V_{D_{1}}{ }^{\prime}(\rho) \\
\hline 0.0 & 4.333 \\
0.5 & 4.318 \\
1.0 & 5.808 \\
1.5 & 8.902 \\
2.0 & 13.599 \\
2.5 & 19.902 \\
3.0 & 27.808
\end{array}
\end{aligned}
$$




$$
D_{2}=\left(\begin{array}{rrr}
1 & 1 & 0 \\
1 & -1 & 0 \\
-1 & 1 & 0 \\
-1 & -1 & 0 \\
0 & 1 & 1 \\
0 & 1 & -1 \\
0 & -1 & 1 \\
0 & -1 & -1 \\
1 & 0 & 1 \\
-0 & 1 & 1 \\
0 & -1 & -1 \\
0 & -1 & 0 \\
0 & 0 & 0 \\
0 & 0 & 0 \\
0 & 0 & 0
\end{array}\right.
$$

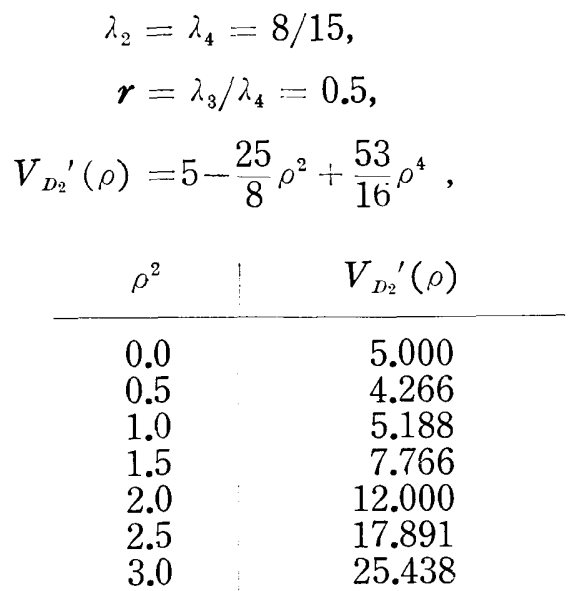

where $D_{1}$ is an usual 3 -factor composite design matrix with elements \pm 1 or 0 , and $D_{2}$ is selected to have $\left(\lambda_{2}, r\right)$ near the values tabulated in Table 2. The mean standardized variances $V_{D_{1}}{ }^{\prime}(\rho)$ and $V_{D_{2}}{ }^{\prime}(\rho)$, for the assigned values of $\rho^{2}=t_{1}^{2}+t_{2}^{2}+t_{3}^{2}$, of the estimated responses are shown above. We observe that,

$$
V_{D_{1}}{ }^{\prime}(\rho)>V_{D_{2}}{ }^{\prime}(\rho), \text { for } 0.2<\rho^{2}<12.2 \text {. }
$$

Moreover, since $D_{1}$ and $D_{2}$ correspond to the absolutely symmetric designs, we can apply the formulae (5.7) and (5.8) for the 3rd order terms which might be contained in the true response $\eta(\mathfrak{X})$. Hence, comparing the coefficient $r$ of $\beta_{i j j}$ in (5.8), we can expect that the bias caused by $D_{2}$ is less than the bias caused by $D_{1}$.

For the second example, we refer to Box-Behnken [2], p. 862, Table 8, 'Seven factor second order rotatable design in three level' of size 66, whose standardized variance is given by

$$
V_{L}(\rho)=0.55\left(12-3 \rho^{2}+8 \rho^{4}\right) \text {. }
$$

Simple calculation shows that, comparing with Table 1 in section 3 , the variance function $V_{D}(\rho)$ in $(6.4)$ is somewhat undesirable. But the modification remains unsolved.

7. Acknowledgement. The author wishes to express his thanks to Prof. Tosio Kitagawa and Asst. Prof. Kazumasa Kôno for proposing this investigation, and to Dr. Eizi Horikawa for preparing Table 2.

\section{References}

[1] G. E. P. Box and T. S. Hunter, "Multi-factor Experimental Designs for Exploring Response Surfaces," Ann. Math. Stat,, Vol. 28 (1957), pp. 195-241.

[2] G. E. P. Box and D. W. Binnken, "Simplex-Sum designs : A Class of Second Order Rotatable Designs Derivable from those of First Order," Ann. Math. Stat., Vol. 31 (1960), pp. 838-864.

General Education Department, Kyushu University. 